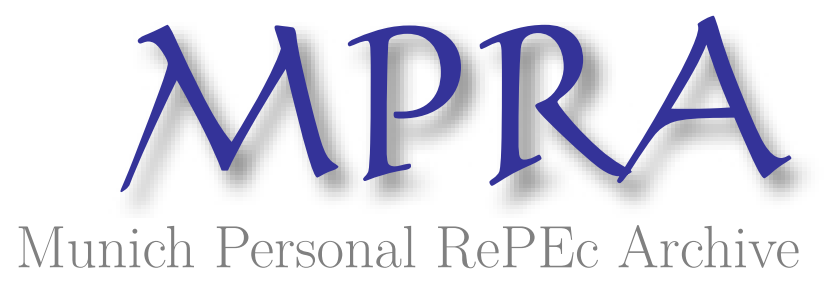

Beyond the transformation problem. Preliminary result of a discussion about the approach of F. Helmedag in the framework of the labour theory of value

Quaas, Friedrun and Quaas, Georg

University of Leipzig

1996

Online at https://mpra.ub.uni-muenchen.de/20529/

MPRA Paper No. 20529, posted 07 Feb 2010 15:23 UTC 


\title{
Jenseits des Transformationsproblems. Vorläufiges Resümee einer Diskussion zum werttheoretischen Ansatz von F. Helmedag
}

\author{
Friedrun Quaas und Georg Quaas, Leipzig
}

Man kann die Ergebnisse des Wissenschaftshistorikers Thomas S. Kuhn auch so interpretieren: der Übergang zu einer neuen wissenschaftlichen Sicht der Dinge erfordert die Kritik der alten wissenschaftlichen Tradition(en) und die Präsentation einer leistungsfähigeren Theorie. Daran scheint sich die Strategie Helmedags zu orientieren, der die neoklassische und die (vor allem an den Namen Sraffas geknüpfte) neoricardianische Tradition durch eine neue ersetzen möchte, in deren Mittelpunkt eine von kurzschlüssigen politischen Auslegungen befreite Werttheorie steht. ${ }^{1}$

Unter diesen Voraussetzungen bedeutet Präsentation einer leistungsfähigeren Theorie die Demonstration der Überlegenheit des werttheoretischen Ansatzes bei der Lösung der ökonomischen Probleme, die von den Vertretern der Neoklassik und von den Neoricardianern zurückgelassen worden sind (oder bisher einfach nicht gelöst werden konnten). Insofern es richtig ist, daß entscheidende Prämissen der Neoklassik bereits durch die grundlegende Arbeit Sraffas entwertet worden sind, ${ }^{2}$ kann sich Helmedag in kritischer Absicht auf die Anomalien der neoricardianischen Theorie konzentrieren. Wir halten Helmedags Kritik nicht für ein Ablenkungsmanöver von den Mängeln seines eigenen Ansatzes, sondern betrachten ihn als notwendigen Bestandteil einer von vornherein verfolgten Strategie. ${ }^{3}$ Richtig bleibt natürlich trotzdem, daß durch die Falschheit anderer Theorien die Richtigkeit der eigenen nicht erwiesen ist.

Als nicht gerade unbedeutendes Nebenprodukt seines Nachweises der Erklärungskraft der Werttheorie offeriert uns Helmedag gleich noch eine Lösung des Transformationsproblems. Bekanntlich handelt es sich dabei um die korrekte mathematische Umwandlung zwischen Wert- und Preisgrößen der Waren, ein Problem, das Marx unerledigt hinterlassen hatte und um dessen Richtigstellung sich unter anderem Ladislaus v. Bortkiewicz verdient gemacht hat.

$1 \mathrm{Zu}$ diesem Aspekt schreibt Helmedag, daß die Wertlehre zwar als „Kampftheorie der Arbeiterklasse“ gelte, sich aber viel eher ableiten ließe, daß sie ,eine Theorie der Leistungsgesellschaft“" sei. (Helmedag, F. (1992), Warenproduktion mittels Arbeit. Zur Rehabilitation des Wertgesetzes, Marburg, S. IX f. Im weiteren zitiert als Helmedag, F., WmA.) Wir erwähnen diesen politischen Aspekt hier nur, um ihn - wie jener Autor - bis zur Klärung grundlegenderer theoretischer Fragen zurückzustellen.

2 Vgl. dazu Helmedag, F., WmA, S. 207 und S. 307 f.

3 Im Gegensatz dazu ist Heinz D. Kurz der folgenden Auffassung: „Anstatt sich Lagers Kritik ... zu stellen, wählt er [Helmedag - d.V.] eine Strategie, die darin besteht, zunächst einen Strauß gänzlich neuer Fragen aufzuwerfen, darunter diejenige nach der Leistungskraft anderer Ansätze als dem seinigen." (Kurz, H.D. (1995), F. Helmedag und die „ökonomische Logik“. In: Jahrbücher für Nationalökonomie und Statistik, Bd. 21, S. 711. Im weiteren zitiert als Kurz, H.D., Logik.) 
Aus der Sicht der neoricardianischen Schule handelt es sich dabei allerdings um ein Scheinproblem: da die Produktionspreise unabhängig von den Werten bestimmt werden können und auf einem bestimmten Niveau der praktischen Entwicklung und der theoretischen Betrachtung zugegebenermaßen die Produktionspreise anstelle der Arbeitswertpreise die Tauschverhältnisse bestimmen, sind die Arbeitswerte überflüssig. ${ }^{4}$ Die neoricardianische Lösung besteht demnach darin, die Ebene der Wertpreise zu eliminieren.

Helmedag präsentiert uns eine diametral entgegengesetzte „Lösung“ des Transformationsproblems, die in der Elimination der Produktionspreise besteht: da die Sraffasche Produktionspreistheorie zu einer Reihe von Anomalien führt, die bisher nicht gelöst werden konnten, 5 kommt Helmedag zu dem Ergebnis, daß eine Preisbildung, die sich nach dem von Sraffa entworfenen Mechanismus richten würde, in der Realität keine Chance hätte. ${ }^{6}$ Dagegen glaubt der Autor von $W m A$, zeigen zu können, daß die Werttheorie einen Verteilungsmechanismus impliziert, der einer dem Konkurrenz- und Leistungsgedanken verpflichteten Wirtschaft entspricht.

Helmedags „Lösung“ des Transformationsproblems besteht also darin, daß zur Abwechslung einmal die Werte die Preise ersetzen sollen. Konsequenterweise muß auch er das Transformationsproblem als ein Scheinproblem betrachten, ${ }^{7} \mathrm{da}$ die Notwendigkeit einer Transformation entfällt, wenn die Produktionspreise irrelevant sind. ${ }^{8}$

Dieser zugegebenermaßen kühne Entwurf hat - vor allem wegen der Angriffe auf die neoricardianische Theorie - zu heftigen Auseinandersetzungen geführt, auf deren sachliche Komponenten im folgenden eingegangen werden soll. Dabei werden wir die Begründung der werttheoretischen Auffassungen Helmedags einschließlich der bisher geäußerten gegnerischen Kritik in den Mittelpunkt stellen - insofern sich die beteiligten Seiten zur Sache äußern. Da wir glauben, daß sich andere Autoren für die Verteidigung der neoricardianischen Theorie

4 Etwas verschämt spricht man in diesem Zusammenhang von der „Redundanz der Mehrwerttheorie“. (Vgl. Fees-Dörr, E. (1989), Die Redundanz der Mehrwerttheorie., Marburg.) Allen Beteiligten dürfte aber klar sein, daß die Werttheorie als Ganzes gemeint ist. In diesem Sinne interpretiert dann auch Helmedag (richtig!) die neoricardianische „Lösung“ des Problems. (Vgl. Helmedag, F., WmA, S. 194 und S. 230 f.)

5 Eine Liste der Anomalien rekapituliert Helmedag in seiner Replik auf Christian Lagers Kritik an seinem Ansatz. (Vgl. Helmedag, F. (1995), Ohne Fleiß kein Preis: Nochmals zur Erklärungskraft der Arbeitswertlehre. In: Jahrbücher für Nationalökonomie und Statistik, Bd. 214, S. 476 f. Im weiteren zitiert als Helmedag, F., Ohne Fleiß.)

6 Vgl. Helmedag, F., WmA, S. 275.

7 Vgl. ebenda, S. 205.

8 Helmedag äußert sich zu diesem Punkt allerdings sehr vorsichtig, indem er „die Wertebene als Ergänzung zum Preis- und Mengensystem“ aus seiner Untersuchung ausklammert. Keinesfalls solle damit Marx' Konzept eingeschätzt werden, der bekanntlich an eine vom Wert abhängigen Preisebene glaubte (vgl. WmA, S. 171). An späterer Stelle wird Marx dann aber doch dafür getadelt, daß er am Produktionspreis festgehalten hat (vgl. WmA, S. 205). 
besser eignen werden, konzentrieren wir uns hier in erster Linie auf die Kritik der werttheoretischen Argumentation Helmedags.

\section{Das Denken in Quotienten}

Eine generelle Kritik, die sowohl die klassische als auch die nachfolgende ökonomische Theorie treffen soll, bezieht sich auf die Orientierung an der Profitrate als Indikator für den wirtschaftlichen Erfolg eines Unternehmens: „Indem Smith den Blick nicht auf den Gewinn insgesamt, sondern auf den Gewinn pro Stück richtet, bahnt er einem Denken in Quotienten statt in Differenzen, d. h. in relativen statt in absoluten Größen, den Weg. Die nachfolgende ökonomische Theorie rückte nämlich einen Quotienten, die Profitrate - den Gewinn pro eingesetzter Kapitaleinheit -, in den Vordergrund und verlor dabei den Gewinn - die Differenz zwischen Erlösen und Kosten - aus den Augen." 9

Der sachliche Unterschied zwischen den zugrunde liegenden Kategorien ist unbestritten. Die Relevanz der Unterscheidung zeigt Helmedag u. a. anhand der mathematischen Modellierung einer Volkswirtschaft, die nur ein einziges Produkt erzeugt - Ricardos Weizenwelt. ${ }^{10}$ Dabei stellt sich heraus, daß die gewöhnlich angenommene Orientierung des Unternehmers an der Profitrate - im Gegensatz zur Orientierung am Gesamtgewinn - das ebenfalls allgemein angenommene unternehmerische Akkumulationsverhalten nicht erklären kann. ${ }^{11}$

Dies ist einer der Gründe, weshalb Helmedag das Konzept der „Distribution des Profits im Verhältnis zum gesamten eingesetzten Kapital" in Frage stellt. Was dabei als „eingesetztes Kapital“" angesehen wird, darin unterscheiden sich außerdem die Lehrmeinungen. ${ }^{12}$

Ob Helmedag selbst dem kritisierten Denken in Quotienten entgehen kann, ob überhaupt ein so strikter Gegensatz zwischen Denken in „Differenzen“, die Gewinne bedeuten, und einem Denken in „Quotienten“, die Profitraten sind, unterstellt werden darf, kann bezweifelt werden. Zunächst setzt der Quotient im Zähler die Bildung jener angeblich vernachlässigten Differenz voraus, denn es ist der Gewinn, der beim „Denken in Quotienten“ in Beziehung zum Kapital gesetzt wird. Das ist zumindest die unternehmensspezifische Sicht, auf die sich auch Helmedag gern beruft: Um die Profitabilität (die Profitrate) zu bestimmen, ist zunächst die Differenz zwischen Erlösen und Kosten zu bilden und diese dann in einem weiteren Schritt in Beziehung zum investierten Kapital zu setzen.

\footnotetext{
9 Helmedag, F., WmA, S. 117.

10 Vgl. ebenda, S. $127 \mathrm{ff}$.

11 Vgl. ebenda, S. 153 f.

12 Vgl. ebenda, S. 195.
} 
Daraus ergibt sich die Profitrate, die dem Kapitalisten anzeigt, ob er sein Geld nicht vielleicht besser anderweitig angelegt hätte, beispielsweise auf dem Geldmarkt - falls dort eine ausreichend hohe Zinsrate angeboten wird.

Helmedag unterstellt für den überwiegenden Teil seines Buches eine andere Sichtweise, die man als volkswirtschaftlich bezeichnen könnte: Er geht von der Existenz einer Profitrate aus (Quotientendenken!) und fragt nach der Bemessungsgrundlage für die Verteilung des gesamtgesellschaftlich erzeugten Gewinns - der Formel „Gewinn = Profitrate $\times$ eingesetztes Kapital“ gemäß. Das eingesetzte Kapital bildet - neben der vorgegebenen einheitlichen Profitrate die Bemessungsgrundlage für den Gewinnanteil des einzelnen Unternehmens.

\section{Alternative Preissysteme}

Für jene „Bemessungsgrundlage“ gibt es - rein theoretisch betrachtet - drei Möglichkeiten: (i) der Gewinn kann nach Maßgabe des Arbeitsaufwandes (der Lohnkosten), (ii) nach Maßgabe des Materialaufwandes (allgemeiner: der Kapitalgüter - oder in Marxschen Termini: des konstanten Kapitals) oder (iii) des insgesamt investierten Kapitals (Lohn- und Materialkosten) verteilt werden. Dies führt zu drei verschiedenen Preisbildungsmechanismen, die man mathematisch formulieren kann und deren Resultat Helmedag als (i) Wertpreise, (ii) Sraffa-Preise und (iii) Bortkiewicz-Preise bezeichnet. Die entsprechenden Preisvektoren ergeben sich als Lösungen der folgenden Gleichungen:

$$
\begin{aligned}
& \mathbf{p}=\mathbf{p A}+\mathbf{a}_{0} w(1+r) \\
& \mathbf{p}=\mathbf{p A}(1+r)+\mathbf{a}_{0} w \\
& \mathbf{p}=\left(\mathbf{p A}+\mathbf{a}_{0} w\right)(1+r)
\end{aligned}
$$

Wie man sieht, unterscheiden sich die Gleichungen darin, welche Kostenbestandteile mit der Profitrate „hochmultipliziert“ werden. ${ }^{13}$ Außerdem besteht nach Helmedag die klassische Auffassung - zumindest im Fall (iii) -darin zu unterstellen, daß das investierte Kapital vorgeschossen werde. ${ }^{14}$ Dagegen macht Helmedag geltend, daß der (gesamtgesellschaftlich) erzeugte Überschuß auch

13 Die ersten beiden Formeln findet man bei Helmedag, F., WmA, S. 249 und S. 219. Die letzte ist eine Verallgemeinerung, die sich aus Bortkiewicz' Wert-Preis-Transformation ergibt, wenn man zu einem nsektoralen System übergeht und die Wertgrößen mit Hilfe der naturalen Struktur des Inputs und Outputs ausdrückt.

Vgl. Helmedag, F., WmA, S. 120. 
dann verteilt werden müßte, wenn Geldvorschüsse keine Rolle spielten - wobei das Einräumen von Zahlungszielen und Krediten als Hilfskonstruktion dienen soll, um dem Leser die Einnahme einer unternehmerischen Sicht zu erleichtern. 15

Alternativ zu den Preissystemen (ii) und (iii) schlägt Helmedag vor, allein die Lohnkosten als Bezugsgröße zu wählen. Dies bedeutet, den auf gesamtwirtschaftlicher Ebene anfallenden Profit auf der Ebene der Unternehmen ,in Proportion zum variablen Kapital zu verteilen." 16 - Sicherlich ist dies zunächst nur eine theoretische Konstruktion - denn der Profit fällt in den einzelnen Unternehmungen an -, aber andererseits eine, deren Resultate sowohl logisch als auch empirisch überprüfbar sind. Deshalb sehen wir in dem bloßen Fakt der Konstruktion keinen ernsthaften Kritikpunkt. ${ }^{17}$

Jener Vorschlag impliziert die Helmedagsche Lösung des Transformationsproblems. Sie läuft darauf hinaus, die Parallelität von Wert- und Preisrechnung zu eliminieren, indem die übliche Methoden der Berechnung der Produktionspreise, die sich entweder allein auf die Materialkosten (Sraffa) oder aber auf die Summe von Material- und Lohnkosten (Bortkiewicz) beziehen, ad absurdum geführt und statt dessen ein alternativer Gewinnverteilungsmechanismus etabliert wird, der die Lohnkosten als Verteilungsmaßstab des Gewinns kreiert. Letzterer wird als werttheoretische Preisbildung deklariert. Damit ist klar, daß sich Helmedag in erster Linie gegen die Produktionspreistheorie in der Marxschen Tradition, die im Fall der kapitalistischen Produktion immer Lohn- und Kapitalkosten berücksichtigte, und die der neoricardianischen Schule richten muß, während die neoklassische Auffassung nur am Rande diskutiert wird.

Unter historischem Gesichtspunkt betrachtet ist es u.a. Bortkiewicz, der angebliche Vater der Lösung des Transformationsproblems, 18 der „,die von Marx (und allen anderen) intendierte Distribution des Profits im Verhältnis zum gesamten eingesetzten Kapital (übernahm - d.V.), ohne gegen diese Verteilungsbasis Bedenken anzumelden."19 Die entsprechende Preisstruktur zeigt Gleichung (iii). Aber schon Marx wußte, daß zumindest die Löhne in der kapitalistischen Realität keineswegs vorgeschossen werden. Wird dies berücksichtigt, gelangt man theoretisch zu einer anderen Bemessungsgrundlage, bei der lediglich die

15 Dies wird besonders deutlich hervorgehoben in Helmedag, F., Ohne Fleiß, S. 479.

16 Vgl. Helmedag, F., WmA, S. 198.

17 Vgl. Lager, Ch., (1995), Über einen weiteren vergeblichen Versuch zur Rechtfertigung der Arbeitswertlehre. Ein Kommentar zu Professor Helmedag. In: Jahrbücher für Nationalökonomie und Statistik, Bd. 214, S. 467. Im weiteren zitiert als Lager, Ch., Kommentar.

18 In Wahrheit hat W. Mühlpfordt bereits 1893 bzw. 1895 eine algebraisch einwandfreie Lösung vorgelegt, die außerdem schon den n-sektoralen Fall behandelt. Vgl. dazu Quaas, F. (1992), Das Transformationsproblem. Marburg. Sowie dieselbe: Wolfgang Mühlpfordt - ein Vorgänger von Bortkiewicz? Zu den theoretischen Quellen des sogenannten Transformationsproblems. In: Jahrbücher für Nationalökonomie und Statistik, Bd. 208 (1991), S. 493-504.

Helmedag, F., WmA, S.195. 
„Materialkosten“ veranschlagt werden. (Siehe Gl.ii!) „Das ist im übrigen die in der modernen Literatur herrschende Sichtweise; deswegen werden wir die so kalkulierten Preise künftig 'Sraffa-Preise' nennen." $20 \mathrm{Da}$ dies nicht nur die Sichtweise Sraffas, ja nicht einmal seine einzige Sichtweise ist, weiß Helmedag, ändert aber nichts an jener etwas einseitigen Bezeichnung der entsprechenden Preise.

\section{Demokratische Entscheidung über Preissysteme?}

Eine Entscheidung zwischen den drei Preissystemen soll nun u.a. dadurch herbeigeführt werden, daß die Profite, die in den drei Sektoren (Produktion von Produktionsmitteln, Lohngütern und Luxusartikeln) je nach dem gewählten Preissystem anfallen, in ihrer Höhe miteinander verglichen werden. ${ }^{21} \mathrm{Da}$ das Wertpreissystem zwei der drei Sektoren begünstigt, schließt Helmedag: „Die Arbeitswerttheorie hätte also die Mehrheit der Kapitalisten hinter sich."22

Hier wird ganz klar in Anlehnung (man beachte den Konjunktiv im obigen Zitat!) an ein demokratie-theoretisches Voten-Modell argumentiert. Ähnlich lesen wir an anderer Stelle: „Wie ersichtlich, hätte die Arbeitswertlehre die Mehrheit der Sektoren hinter sich. Doch sie darf nicht nur deswegen Überlegenheit beanspruchen." 23 - Es mag ja sein, daß die Überlegenheit der Werttheorie noch anders gezeigt werden kann, hier aber wird klar einer der Gründe benannt. Unverständlich ist deshalb, warum Helmedag seinen Kritiker tadelt, wenn dieser ihm den Schluß vom Mehrheitsvotum der Sektoren auf die Überlegenheit der Werttheorie unterstellt. ${ }^{24}$

Dieses methodische Vorgehen wurde von Christian Lager in der anschließenden Diskussion für fragwürdig gehalten, weil die Entscheidung zwischen alternativen Preissystemen niemals auf sektoraler Ebene getroffen wird. „Entscheidungsträger sind jene Akteure, die über die Art und das Ausmaß der Produktion, also über die Technikwahl und den sektoralen Kapitaleinsatz entscheiden." 25

Streng genommen entscheiden aber auch diese Akteure nicht über Preissysteme, sondern bestenfalls über ihre Angebotspreise, die aber mit den theoretisch konstruierten Produktionspreisen wenig zu tun haben. Offenbar handelt es sich bei Helmedag um den Versuch, die Akzeptanz unterschiedlicher Preissysteme aus

20 Vgl.ebenda

21 Vgl. ebenda, S. $200 \mathrm{f}$.

22 Ebenda.

23 Helmedag, $F$ (1993) Zur Berechtigung der grundlegenden theoretischen Konstruktion von Marx im ersten Band des „Kapital“. In: Jahrbücher für Nationalökonomie und Statistik, Bd. 212, S. 449.

24 Vgl. Helmedag, F., Ohne Fleiß, S. 480.

25 Lager, Ch., Kommentar, S. 467. 
der Sicht unterschiedlicher sektoraler Interessenlagen, die durchaus auch auf die Einzelunternehmen durchschlagen, auszuloten. Daß er dabei auf das Modell demokratischer Abstimmung anspielt, kann leicht mißverstanden werden, auch wenn explizit betont wird, daß es sich realiter um einen andersartigen Prozeß handelt. ${ }^{26}$ Dieses Problem wird auch nicht dadurch beseitigt, daß sich Helmedag in seiner Replik der entsprechenden Kritik Lagers von einem Voten-Modell auf ein Veto-Modell zurückzieht. ${ }^{27}$

Vermutlich im Gefühl, daß Helmedags methodisches Vorgehen vielleicht doch nicht ganz danebengreifen könnte, läßt sich Christian Lager auf dessen Argumentationsweise ein und erörtert die Frage, ob der durch das Wertpreissystem benachteiligte Sektor I gezwungen werden kann, ein ihm unangenehmes Preissystem zu akzeptieren. Dabei begeht dieser Kritiker einen weiteren groben Schnitzer: den Inhalt des Buches WmA, das als ausführliche Darstellung des Helmedagschen Anliegens betrachtet werden muß, offenbar nicht zur Kenntnis nehmend, behauptet Lager irrtümlicherweise, daß die Technikwahl nicht thematisiert werde und deshalb die Frage nach der Profitabilität der Übernahme der Produktion der Produktionsmittel durch die Unternehmer der Sektoren II und III von Helmedag nicht begründet beantwortet werden konnte. ${ }^{28}$ Letzterer hat hier keine Mühe, diese Kritik als freie Erfindung zurückzuweisen. 29

\section{Helmedags technologieabhängige Voten}

Diese Mißgriffe verdunkeln etwas jene Seiten der Kritik Lagers, die u. E. hinreichen, um Helmedags „demokratie-theoretische“ Argumentation umzustoßen. Ungeschickterweise hat Lager diese Kritik auch noch in einer Fußnote versteckt. Sie lautet:

„Ändert man die Technologie des Sektors 2 derart, daß dieser an Stelle von 100 Arbeitseinheiten konstantes Kapital nunmehr 300 Arbeitseinheiten einsetzt, so zeigt sich, wie kleinere Kalkulationen beweisen, daß plötzlich die 'Mehrheit' der Sektoren für das 'Sraffa'-Preissystem votierte." 30

Bedeutet dies, daß Helmedags allgemein-theoretisches Plädoyer für das Wertpreissystem allein auf der „geschickten“ Wahl seines Beispiels beruht? Es sollte sich lohnen, dieser Frage nachzugehen und den Hinweis Lagers etwas ausführli-

26 Vgl. Helmedag,, F., WmA, S. $200 \mathrm{f}$.

27 Dieses lautet dann: „Egal welche Zahlen und wieviel Zweige man nimmt, den Bortkiewicz- oder den SraffaPreisen würde stets mindestens einer die Zustimmung verweigern: die Eigenherstellung der Vorprodukte wäre für ihn lukrativer.“ (Helmedag, F., Ohne Fleiß, S. 480.)

28 Vgl. Lager, Ch.,, Kommentar, S. 467.

29 Vgl. Helmedag, F., Ohne Fleiß, S. 474.

30 Lager, Ch., Kommentar, S. 466, Fn. 8. 
cher darzustellen, zumal Helmedag in seiner Replik mit keinem Wort darauf eingeht und Lager selbst sich in diesem Punkt zwar einen Sieg bescheinigt, der aber ohne konkreten Nachweis nicht einsichtig ist. ${ }^{31}$

Helmedag rechnet mit folgendem Wertpreisschema, das eine einheitliche Mehrwertrate von $2 / 3$ unterstellt:

\begin{tabular}{|c|c|c|c|c|}
\hline Abteilung & $\begin{array}{c}\text { Konstantes } \\
\text { Kapital }\end{array}$ & $\begin{array}{c}\text { Variables Ka- } \\
\text { pital }\end{array}$ & Mehrwert & Produktwert \\
\hline I & 225 & 90 & 60 & 375 \\
\hline II & 100 & 120 & 80 & 300 \\
\hline III & 50 & 90 & 60 & 200 \\
\hline I-III & 375 & 300 & 200 & 875 \\
\hline
\end{tabular}

Lager schlägt für den Sektor II eine Technologieänderung vor, bei der die 100 AE (Arbeitseinheiten) konstantes Kapital durch 300 AE ersetzt werden. Läßt man alle anderen Größen unverändert, gerät das System aus dem Gleichgewicht, wie das Einsetzen der entsprechenden Werte zeigt:

\begin{tabular}{|c|c|c|c|c|}
\hline Abteilung & $\begin{array}{c}\text { Konstantes } \\
\text { Kapital }\end{array}$ & $\begin{array}{c}\text { Variables Ka- } \\
\text { pital }\end{array}$ & Mehrwert & Produktwert \\
\hline I & 225 & 90 & 60 & 375 \\
\hline II & 300 & 120 & 80 & 500 \\
\hline III & 50 & 90 & 60 & 200 \\
\hline I-III & 575 & 300 & 200 & 1075 \\
\hline
\end{tabular}

Der Bedarf an Produktionsmitteln kann durch den Sektor I nicht mehr gedeckt werden. Auf der anderen Seite entsteht ein Überschuß an Lebensmitteln im Werte von $200 \mathrm{AE}$. Um das Gleichgewicht wieder herzustellen, versehen wir Sektor I und III mit je einem Multiplikator, der garantiert, daß die technologische Struktur bei geändertem Aktivitätsniveau erhalten bleibt: 32

31 Vgl. Lager, Ch. (1995), Auch mit viel Fleiß - kein Preis oder Einstimmigkeit ist nicht leicht zu erzielen. Eine Antwort auf Professor Helmedag. In: Jahrbücher für Nationalökonomie und Statistik, Bd. 214, S. 730 f. Im weiteren zitiert als Lager, Ch.,, Einstimmigkeit.

32 Der Faktor 5/3 ist die Summe von $1+\mathrm{m}^{\prime}$, mit der das variable Kapital zu multiplizieren ist, um das Marxsche Wertprodukt (Neuwert) zu erhalten. 
$225 \alpha+\frac{5}{3} 90 \alpha=375 \alpha$

$300+\frac{5}{3} 120=500$

$50 \beta+\frac{5}{3} 90 \beta=200 \beta$

Dieses Gleichungssystem hat eine eindeutige Lösung bei $\alpha=23 / 9$ und $\beta=5 / 3$. Das von Lager vorgeschlagene Wertpreisschema - die $m^{\prime}$-Preise in der Diktion Helmedags - lautet demnach (hier - wie in den folgenden Tabellen - auf Zehntel gerundet):

\begin{tabular}{|c|c|c|c|c|}
\hline Abteilung & $\begin{array}{c}\text { Konstantes } \\
\text { Kapital }\end{array}$ & $\begin{array}{c}\text { Variables Ka- } \\
\text { pital }\end{array}$ & Mehrwert & Produktwert \\
\hline I & 575 & 230 & 153,3 & 958,3 \\
\hline II & 300 & 120 & 80 & 500 \\
\hline III & 83,3 & 150 & 100 & 333,3 \\
\hline I-III & 958,3 & 500 & 333,3 & 1791,7 \\
\hline
\end{tabular}

Rechnet man jetzt wie gewöhnlich nach dem Bortkiewicz-Verfahren in Preise um, erhält man die von Helmedag so bezeichneten $\rho$-Preise:

\begin{tabular}{|c|c|c|c|c|}
\hline Abteilung & $\begin{array}{c}\text { Konstantes } \\
\text { Kapital }\end{array}$ & $\begin{array}{c}\text { Variables Ka- } \\
\text { pital }\end{array}$ & Profit & Produktwert \\
\hline I & 690 & 276 & 184 & 1150 \\
\hline II & 360 & 144 & 96 & 600 \\
\hline III & 100 & 180 & 53,3 & 333,3 \\
\hline I-III & 1150 & 600 & 333,3 & 2083,3 \\
\hline
\end{tabular}

Hierbei wurde der Profit auf die Summe des konstanten und variablen Kapitals berechnet. Die folgende Tabelle enthält die sog. $r$ - oder Sraffa-Preise, bei denen allein die „Materialkosten“ die Bemessungsgrundlage für den anfallenden sektoralen Profit darstellen: 


\begin{tabular}{|c|c|c|c|c|}
\hline Abteilung & $\begin{array}{c}\text { Konstantes } \\
\text { Kapital }\end{array}$ & $\begin{array}{c}\text { Variables Ka- } \\
\text { pital }\end{array}$ & Profit & Produktwert \\
\hline I & 750 & 300 & 200 & 1250 \\
\hline II & 391,3 & 156,5 & 104,4 & 652,2 \\
\hline III & 108,7 & 195,6 & 29,0 & 333,3 \\
\hline I-III & 1250,0 & 652,1 & 333,433 & 2235,5 \\
\hline
\end{tabular}

Wir folgen der Methode Helmedags ${ }^{34}$ und stellen die sektoralen Profite alternativer Preissysteme in einer Tabelle zusammen:

\begin{tabular}{|c|c|c|c|}
\hline Abteilung & $\rho$-Preise & r-Preise & m'-Preise \\
\hline I & 184 & $\mathbf{2 0 0}$ & 153,3 \\
\hline II & 96 & $\mathbf{1 0 4 , 4}$ & 80 \\
\hline III & 53,3 & 29,0 & $\mathbf{1 0 0}$ \\
\hline I-III & 333,3 & 333,4 & 333,3 \\
\hline
\end{tabular}

Wie bei Helmedag sind die maximalen Profite kursiv und fett gedruckt worden. Man erkennt leicht, daß bei diesem Beispiel die Sektoren I und II das SraffaPreissystem präferieren, während nur ein einziger Sektor hinter den Wertpreisen Helmedags steht. Mit anderen Worten: Lager hat recht, wenn er behauptet, daß Helmedags Argumentation auf einer zufällig gewählten Technologiestruktur beruht. Aufgrund der nach Lagers Kritik eingeführten abgeschwächten Variante des Helmedagschen Entscheidungsverfahrens würde sogar gelten: alle Preissysteme haben - übrigens auch schon in Helmedags ursprünglichem Beispiel mindestens ein Veto gegen sich - m. a. W.: durch das Veto-Modell kann überhaupt keine Entscheidung herbeigeführt werden. Lagers Kritik an der Reformulierung des Entscheidungsverfahrens ${ }^{35}$ ist deshalb noch um einen Schritt zu verschärfen: die Methode spricht nicht nur gegen die Helmedag-Preise, sondern ist generell als Entscheidungsverfahren nicht zu gebrauchen.

33 Die 4 nach dem Komma anstelle einer erwarteten 3 kommt durch die Rundung auf Zehntel zustande.

34 Vgl. Helmedag, F., WmA, S. 200.

35 Vgl. Lager, Ch, Einstimmigkeit, S. 731. 


\section{Angebliches Versagen der Bortkiewicz- und der Sraffa-Preise}

Allerdings werden von Helmedag weitere Argumente beigebracht: BortkiewiczPreise und Sraffa-Preise lassen sich in einem dreisektoralen Modell nicht berechnen, wenn das konstante Kapital des Lohngütersektors gleich Null ist. ${ }^{36}$ Helmedag wertet dies als Versagen der entsprechenden Preismodelle, sieht zugleich aber ein gewichtiges Gegenargument voraus: „Der Einwand liegt nahe, die gerade analysierte kapitallose Produktion der Abteilung II tauche in der Wirklichkeit kaum auf." 37 Trotzdem sei kapitallose Produktion ein Sonderfall, der die allgemeine Geltung der Theorie widerlege. 38

Hier ist offenbar eine wissenschaftstheoretische Argumentation angebracht. Vom Standpunkt der kritisch-rationalistischen Wissenschaftstheorie müßte man verschärfend sagen, daß durch ein Gegenbeispiel nicht nur die allgemeine Geltung der Theorie, sondern die Theorie selbst widerlegt wird. Vorausgesetzt ist dabei, daß es sich um ein empirisches Gegenbeispiel handelt. Dies ist hier aber nicht der Fall: die von Helmedag konstruierte kapitallose Produktion ist, vom Standpunkt derselben philosophischen Richtung, reine Metaphysik - wie die Behauptung der Existenz von Pegasus: ein entsprechendes Objekt kann nicht nachgewiesen werden.

Man tut gut daran, metaphysische Beispiele nicht als falsifizierende Instanz empirischer Theorien gelten zu lassen. Man könnte sonst auch argumentieren, daß das Kopernikanische Weltmodell im Fall einer Sonnenmasse gleich Null versagt: in diesem Fall hätte unser Sonnensystem keine Planeten. Es hat aber welche, sollen wir daraus schließen, daß das Kopernikanische Weltmodell falsch ist? Oder ein anderes Beispiel: Eine der am besten bewährten Theorien, die Maxwellsche Elektrodynamik, versagt für räumlich verschwindend kleine Ladungen. Trotzdem denkt wohl kein Physiker ernsthaft daran, auf diese Theorie zu verzichten.

Nun sind räumlich verschwindende Ladungen oder eine Sonne mit der Masse Null sicher genauso empirisch unsinnige Annahmen wie eine Warenproduktion ohne konstantes Kapital. Daraus allein läßt sich jedenfalls nicht auf die Ungültigkeit der Bortkiewicz- oder Sraffa-Preise schließen. Empirische Theorien können nicht widerlegt werden, indem man zeigt, daß sie in Fällen, die empirisch nicht vorkommen, versagen.

Doch wir wollen nicht mißverstanden werden: Helmedag diskutiert in seinem Buch eine Reihe von Eigenschaften und Konsequenzen der neoricardianischen Theorie, die durchaus empirisch relevant sind und der ökonomischen Realität

36 Vgl. Helmedag, F., WmA, S. $201 \mathrm{ff}$. und $251 \mathrm{ff}$.

37 Ebenda, S. 203.

38 Vgl. ebenda. Dabei beruft sich Helmedag auf den in dieser wissenschaftstheoretischen Frage völlig unmaßgeblichen Bortkiewicz. 
des Konkurrenzkapitalismus zu widersprechen scheinen - dieser Eindruck entsteht jedenfalls schon bei der Analyse der logischen Mängel, auf die sich Helmedag konzentriert. Die Bortkiewicz-Preise fallen bei der obigen Argumentation gleich mit unter den Tisch, obwohl gegen sie kaum mehr als das verunglückte Voten-Modell und die Falsifikation unter empirisch irrelevanten Bedingungen spricht.

\section{Kapitalwanderung als Entscheidungsmechanismus}

Bevor wir auf die grundsätzliche Kritik Helmedags an der neoricardianischen Theorie weiter eingehen, die in gewissem Maße auch andere Theorien treffen könnte, möchten wir die Frage, welche Mechanismen denn nun tatsächlich eine Entscheidung zwischen den Preissystemen herbeiführen, zum Abschluß bringen. Wie bereits bemerkt, spielt das Voten-Modell nur die Rolle einer Hilfskonstruktion. Nachdem gezeigt worden ist, daß es nicht einmal in der Lage ist, Helmedags eigene Sichtweise allgemeingültig zu belegen, können wir es wohl vergessen. An die Stelle der „demokratie-theoretischen“ Argumentation tritt somit die ökonomische Vorstellung der Kapitalwanderung unter der Bedingung konstanter Skalenerträge und freien Zugangs zu allen Produktionsmethoden.

Angenommen, den Unternehmern des Sektors II und III paßt das Produktionspreissystem nicht, das ihnen der Sektor I aufdrücken will. Aufgrund ihres arbeitswerttheoretischen Instinkts wittern sie eine bodenlose Ungerechtigkeit, wenn der Überschuß durch einen Preis verteilt wird, der die technikintensive Produktion von Produktionsmitteln begünstigt. „Sie kennen den 'aktuellen' Wert der Produktionsmittel und prinzipiell hält nichts und niemand die Sektoren II und III davon ab, sich die nötigen Produktionsmittel in einem Parallelprozeß selbst zu verschaffen. Die verfügbare Arbeitskraft muß lediglich betriebsintern in geeigneten Proportionen mit der Fabrikation der Inputgüter betraut werden. Notfalls erzeugen Abteilung II die Luxuswaren und Abteilung III die Lohngüter in jeweils eigener Regie, die Wirtschaft löst sich in autarke Produktionsinseln auf". 39

Dies ist also der tiefere Grund, weshalb die kapitalistische Wirtschaft von Wertpreisen beherrscht sein soll: „Wegen der Möglichkeit der alternativen Eigenfertigung ти $\beta$ im Gleichgewicht die allgemeine Profitrate mit der Mehrwertsrate übereinstimmen. Wäre die Profitrate geringer, scherte zumindest eine Abteilung aus der Arbeitsteilung aus. Die Sektoren können nur dann erwarten, Abnehmer ihrer Produkte zu finden, wenn die Tauschrelationen den Werten ent-

39 Ebenda, S. 204. 
sprechen; das ist die einzige, auf einmütige Billigung der Unternehmerkollegen stoßende Methode, das Mehrprodukt als Profit 'richtig' aufzuschlüsseln. “40

Doch unglücklicherweise kennen weder Helmedag noch seine Unternehmer die Wertpreise. Und selbst wenn diese ihnen bekannt wären - ihre Geldgeber oder ihr kapitalistisches Eigeninteresse würden verhindern, ausgerechnet die Arbeitsinputs als gerechte Basis der Verteilung des Überschusses betrachten zu wollen. Das den kapitalistischen Verwertungsbedingungen entsprechende Analogon zu Helmedags Argumentation würde vielmehr lauten: Wegen der Möglichkeit der alternativen Eigenfertigung $т и \beta$ im Gleichgewicht die sektorale Profitrate mit der allgemeinen Profitrate - berechnet auf die Summen der Kosten von Lohn- und Kapitalgütern - übereinstimmen. Wäre die Profitrate geringer, scherte zumindest eine Abteilung aus der Arbeitsteilung aus. Könnte zum Beispiel die Lobby der Produktionsmittelhersteller den Preis nicht durchsetzen, der für eine Verzinsung der in Sektor I zeitweilig gebundenen Werte erforderlich ist, wanderte das Kapital in die Sektoren II und III ab.

Wie man sieht, läßt sich Helmedags Argumentation für wertadäquate Preise ohne Probleme (und u. E. sogar plausibler) auch für den Produktionspreis verwenden. Allerdings bleibt in beiden Fällen unklar, wodurch der Kapitalwanderung Grenzen gesetzt sind. Es ist eine bloße Unterstellung, daß die Kapitalwanderung $\mathrm{zu}$ einer Integration der verschiedenen Produktionsmethoden führen würde. Genauso denkbar ist, daß das zunächst in einem Sektor frei werdende Kapital sich ganz auf die Produktion in einem anderen Sektor wirft. Das dadurch gemindert Angebot beispielsweise an Produktionsmitteln würde in der Folge eine Erhöhung des Preises für die Produkte des Sektors I erzwingen, und zwar bis zu dem Punkt, an dem auch dort eine profitable Produktion möglich wird.

Ergänzt man die Argumentation Helmedags also durch die realistischen Annahmen, daß die Kapitalwanderung ein Prozeß ist und daß das Verhältnis von Angebot und Nachfrage einen Einfluß auf die Preisbildung und damit auf die Herstellung des Gleichgewichts hat, so landen wir letztlich geradezu zwangsläufig bei den gewöhnlichen Produktionspreisen.

Man fragt sich, wie Helmedag auf den Gedanken einer für jeden Unternehmer auch unabhängig vom Preissystem durchschaubaren Bemessungsgrundlage für die Verteilung des gesamtgesellschaftlich erzeugten Überschusses kommen konnte. Wir vermuten, daß der tiefere Fehler der Konzeption Helmedags darin besteht, die Arbeit als einen exogenen Faktor zu betrachten. ${ }^{41}$ Der Schein, daß die Arbeit unabhängig von der ökonomischen Bewertung durch Wert- oder Produktionspreise in die Kalkulationen eingehen könnte, entsteht möglicher-

40 Ebenda, S. 205.

41 In dem von Helmedag benutzten Gleichungssystem für die Preise erscheint die Arbeit als exogener Faktor. (Vgl. Helmedag, F., WmA, S. 315.) Wohl deshalb spricht er von der Arbeit als einem „originären Faktor“ (vgl. ebenda, S. 247) oder von der ,einzig nicht-produzierten Ressource“ (vgl. ebenda., S. 313). 
weise durch die Abstraktion von der Heterogenität der Arbeit. ${ }^{42}$ Berücksichtigt man diesen Fakt, wird sofort eins klar: ökonomisch relevant ist nicht die Arbeit schlechthin, sondern die ökonomisch bewertete Arbeit. Und diese Bewertung erfolgt durch ein wie auch immer geartetes Preissystem.

Damit verschwindet die Arbeit - wie übrigens alle anderen naturalen Faktoren auch - als exogener Faktor aus den Bewertungsgleichungen und wird zu einer endogenen Größe, die sich mit der Art des theoretisch gewählten Preissystems ändert: die letzte Bastion für eine wert- bzw. preisunabhängige Verteilung des Überschusses ist damit gefallen.

\section{Helmedags grundsätzliche Kritik an Sraffa: Die Rolle der Unternehmerkredite}

In der Identifikation der Bemessungsgrundlage für die Verteilung des Profits mit den Materialkosten sieht nun Helmedag einen der grundlegendsten Konstruktionsfehler der neoricardianischen Theorie. ${ }^{43} \mathrm{Um}$ diesen aufzudecken, werden gleich mehrere Aspekte beleuchtet.

Die erste Argumentationslinie richtet sich gegen die Annahme, daß der Materialaufwand vorzufinanzieren wäre. Dagegen spräche, daß sich ,die Unternehmer einander regelmäßig ein Zahlungsziel“" einräumen.44 Führt man diesen Gedanken etwas aus, ergeben sich folgende Zusammenhänge:

Der Unternehmer A kreditiert als Lieferant seinem Abnehmer B den zu zahlenden Preis des Produkts. Geht letzterer darauf nicht ein und zahlt bei Lieferung, kann er die Preissumme skontieren. Umgekehrt kann ein Skonto als Zinsaufschlag im Fall der Inanspruchnahme des Kredits interpretiert werden. Der Unternehmer B zahlt dann nicht nur für die erworbenen Produkte, sondern auch noch die Kosten des kurzfristigen Kredits, den ihm sein Lieferant A gewährt hat. $\mathrm{Da}$ dies regelmäßig geschieht, ändert nichts an diesem Vorgang. Bedenkenswert ist dagegen, daß der Unternehmer B in einer anderen Beziehung, nämlich gegenüber seinem Abnehmer $\mathrm{C}$, ebenfalls als Lieferant und eventueller Kreditgeber auftritt. Man könnte meinen, daß er hier für den Zins entschädigt wird, den er an A gegebenenfalls zahlen mußte: $B$ kreditiert $C$ und erhält dafür einen Zinsertrag. Auf den ersten Blick betrachtet könnte es deshalb sein, daß sich die gegenseitigen Zinsforderungen der Kapitalisten tendenziell ausgleichen und nach allen gegenseitigen Aufrechnungen der Unternehmer untereinander keine Zins- oder Profiterwartungen übrigbleiben, die sich an die Kapitalgüter knüpfen, d.h., daß die Wertpreisgleichung (i) gilt.

\footnotetext{
42 Auf dieses Problem geht Helmedag nur sehr kurz ein. (Vgl. Helmedag,, F., WmA, S. 325.)

43 Vgl. Helmedag, F., WmA, S. $207 \mathrm{ff}$.

44 Vgl. ebenda, S. 197.
} 
Christian Lager hat versucht, diese Vorstellung in ein Drei-Sektoren-Modell umzusetzen. Dabei nimmt er an, daß die Produktionsmittel erst am Ende einer Periode bezahlt werden müssen, in der sie bereits verwendet worden sind. Dagegen sind Lohn- und Luxusgüter bei Lieferung zu zahlen. Lagers Formalisierung zeigt, daß dieses Modell folgende Alternative impliziert: Entweder verzichten die Produktionsmittelhersteller für eine ganze Periode einseitig auf die Verzinsung ihres Kapitals (sie sind dann - nach Lager - „Philanthropen oder Dummköpfe“), oder es gilt nach wie vor das Bortkiewicz-Preismodell. 45

In seiner Entgegnung zitiert Helmedag aus einem Brief an H. D. Kurz, in dem es in diesem Zusammenhang heißt, daß es ihm ,nicht ganz verständlich [ist], weshalb nur der Sektor I Zahlungsziele in einer 'Urperiode' einräumen soll." "46 _ Diese Antwort ist vieldeutig, denn es bleibt offen, welcher der anderen Sektoren ebenfalls Zahlungsziele einräumt. Die Logik der Argumentation scheint u. E. zu implizieren, daß bei jeder partiellen Kombination der Sektoren dieser Einwand erneut erhoben werden kann. Denkt man das Argument jedoch zu Ende, ergibt sich eine eindeutige Interpretation: Alle Sektoren räumen sich gegenseitig ein Zahlungsziel und damit einen Lieferantenkredit ein, und zwar regelmäßig, d.h. in jeder Periode.

Auf diese ökonomische Interpretation der Gleichung (i) hat bereits Kurz geantwortet, wenn er feststellt, daß sie unlogisch ist, ,da es schlicht nicht möglich ist, daß Verkäufer bereits heute erhalten, was die Käufer erst in Zukunft zahlen.“47 Verbal bezieht Kurz dieses Argument zwar auf die „Zahlung der materiellen Inputs", wozu Lohn- und Luxusgüter wohl nicht gehören; aber die Bezugnahme auf Gleichung (i) macht deutlich, daß er den Fall meint, bei dem alle Produkte kreditiert werden sollen.

Hier könnte Helmedag immer noch entgegnen, daß sowohl Lager als auch Kurz unverbesserlich einem Denken in Perioden aufgesessen sind. Bevor wir jedoch untersuchen, ob die Wertpreisgleichung beim Übergang zu einer dynamischen Betrachtung dem Vorwurf, in sich widersprüchlich zu sein, entgehen kann, analysieren wir noch die Vorstellung, daß sich die gegenseitigen Zinsforderungen aufheben.

Eine solche Auffassung übersieht die quantitativen Unterschiede, die durch das jeweils gewährten Kreditvolumen und die im einzelnen beanspruchten Zahlungsfristen entstehen: Die Zinsbelastung ist - auch bei einem einheitlichen Skontosatz - für die einzelnen Kapitalisten unterschiedlich hoch und schlägt sich deshalb auch in unterschiedlichen Kosten nieder. Auch bei gegenseitiger Kreditierung der Unternehmer gleichen sich die unterschiedlich großen Zinsforderungen nicht aus, sondern führen an den stärker technikbelasteten Punkten

\footnotetext{
45 Lager, Ch., Kommentar, S. $468 \mathrm{f}$.

46 Helmedag, F., Ohne Fleiß, S. 475.

47 Kurz, H.D., Logik, S. 24.
} 
dazu, daß beispielsweise zum Erwerb der Produktionsmittel mehr Geld vorgeschossen oder geborgt werden muß als in weniger technikintensiven Bereichen was entsprechende Zinserwartungen oder -kosten auslöst. Klarerweise muß dies auch in einer volkswirtschaftlichen Kostenrechnung berücksichtigt werden - wie klein dieser Posten im Durchschnitt auch sein mag.

Es gibt aber noch eine prinzipiellere Kritik an dieser Verquickung zwischen der Profiterwartung und dem Problem der mehr oder weniger erforderlichen Vorfinanzierung: Schon in praktischer Hinsicht muß der Profit grundsätzlich vom Zins unterschieden werden, auch wenn beide Kategorien auf die Bindung von sonst als Geld frei verfügbaren Werten zurückzuführen ist. In diesem Punkt stimmen wir mit Helmedag überein. 48 Nimmt man seine Kritik am Quotientendenken ernst und fixiert für einen Moment das unternehmerische Denken in Differenzen, kommt man jedoch zu einer abweichenden Auffassung: Sowohl für den einzelnen Unternehmer als auch für die gesamte Unternehmerschaft steht der Profit notwendigerweise in Beziehung zum eingesetzten Kapital - wie immer sich dieses auf Löhne, Materialien, Arbeitsmittel oder sonstige Belastungen (darunter auch durch die Zinskosten für eventuelle Kredite) verteilen möge. Das Verhältnis des Profits zum eingesetzten Kapital ergibt die Profitrate als Indikator für die Effizienz des eingesetzten Kapitals. Die Frage, was bei gegebener Profitrate - die Bemessungsgrundlage des Profits sei, ist rein akademischer Natur. Alle gegenseitigen Kreditierungen können nichts daran ändern, daß der Unternehmer letztlich nicht nur die Löhne, sondern auch die Investitionsgüter (,Materialkosten“) bezahlen muß. In klassischer Sicht stellt dies die Summe von Kapital- und Lohnkosten dar. An dieser Summe ist ein eventueller Überschuß des Erlöses, d.h. der Profit, zu bemessen. Argumentiert man aus dieser betriebswirtschaftlichen Sicht, sind es somit gerade die Bortkiewicz-Preise, die bei konstantem Reallohn der Realität am nächsten stehen, auch wenn weder Helmedags noch Lagers Beispiel dies deutlich zu machen vermögen.

\section{Der Übergang zur dynamischen Betrachtung}

Die zweite Argumentationslinie Helmedags stützt sich auf den Unterschied zwischen einer statischen und einer dynamischen Betrachtung des ökonomischen Reproduktionsprozesses. Der neoricardianischen Theorie wird vorgeworfen, daß sie die Produktionsperiode in einer Art bäuerlichen Sichtweise mit dem Jahr

48 In der folgenden Aussage wird zwischen beiden Kategorien klar unterschieden: „Mein Anliegen war es aufzuspüren, welche Bemessungsgrundlage des Profits (nicht des Zinses!) ein Gleichgewicht stiftet.“ (Helmedag, F., Ohne Fleiß, S. 479.) 
identifiziert. Gefordert ist dagegen die Betrachtung von Stromgrößen der Volkswirtschaft. 49 „In einer Wirtschaft, in der nicht nur Büroklammern gebogen und Tageszeitungen gedruckt, sondern auch Wolkenkratzer errichtet und Schiffe auf Kiel gelegt werden, ist jede Produktionsperiode ein willkürliches Konstrukt. Infolgedessen ist man berechtigt, bei der Wahl einer uniformen Produktionsperiode die klassische Sichtweise aufzugeben: warum nicht ein Tag, eine Stunde und - im Grenzfall - eine 'logische' Sekunde?"50

Die statische Betrachtung der Volkswirtschaft als ein Ensemble von Bestandsgrößen korrespondiert mit der Auffassung, daß das Kapital (zumindest für das „Material“) vorgeschossen werden muß - die Finanzierung der Bestände. Hier überschneiden sich beide Argumentationslinien und eröffnen eine dritte Dimension, die der Fixkosten nämlich, die nach Helmedag bei einer dynamischen Betrachtungsweise der Ausdruck des Verbrauchs von Kapitalgütern sind. Betrachten wir zunächst, wie eine dynamische Betrachtung zu realisieren wäre!

Stromgrößen werden in der Mathematik durch Differentiale erfaßt, die als Grenzwerte von Differenzen definiert werden. Bei einer differentialanalytischen Betrachtung der Volkswirtschaft unter den Gesichtspunkten Lohnkosten (variables Kapital), Materialkosten (konstantes Kapital) und Erlös (realisierter Wert) wählt man immer kürzere Produktionsperioden, bildet für End- und Anfangspunkt der gewählten Periode die Differenz oder/und den Quotienten der betrachteten Bestandsgrößen und untersucht, wie sich diese Größen und ihre Relationen zueinander beim Übergang zu einer verschwindend kleinen Periode verhalten. Wenn wir von statistischen Schwankungen, die bei Betrachtung immer kleinerer Produktionsperioden immer stärker in Erscheinung treten, einmal absehen und stetige Verläufe für die betrachteten Variablen unterstellen, haben wir bei jeder noch so kleinen Produktionsperiode Kosten für die verbrauchten Materialien (einschließlich Investitionsgüter) sowie für die Löhne der Beschäftigten, und es wird immer einen noch so kleinen Erlös geben, der jene Kosten zu decken hat. Natürlich werden diese Größen bei Betrachtung immer kürzerer Perioden immer kleiner und nähern sich dem Grenzwert Null. Es gibt aber keinen Grund, anzunehmen, daß eine der drei Größen sich mit Verkürzung der betrachteten Perioden ,schneller“ dem Grenzwert Null annähern würde als die anderen. ${ }^{51}$ Dies müßte aber der Fall sein, wenn man annehmen wollte, daß bei einer reinen differentiellen Betrachtung eine der genannten Größen im Verhältnis zu den anderen wegfiele.

49 Um Mißverständnissen vorzubeugen, sei angemerkt, daß Helmedag den Begriff der Stromgröße hier in einem etwas anderem Sinne verwendet als es in der volkswirtschaftlichen Gesamtrechnung üblich ist, wenn Stromgrößen als an eine Zeitperiode gebunden definiert werden. (Vgl. etwa Woll, A. (1993)., Allgemeine Volkswirtschaftslehre, 11. Aufl., München, S. 315.) Im folgenden werden wir versuchen, Helmedags Vorstellung einen mathematisch exakten Sinn zu geben.

50 Helmedag, F., WmA, S. 215.

51 Exakter gesprochen müßte man sagen, daß eine der Größen unendlich klein von höherer Ordnung als die der anderen ist. (Vgl. Smirnow, W.I., (1973), Lehrgang der höheren Mathematik, Teil I, Berlin, S. 88 f.) 
Wir sind deshalb der Meinung, daß auch nach Übergang zu einer Stromgrößenbetrachtung es der Fall sein wird, daß ständig Geld für Material und Investitionsgüter ausgegeben werden muß und daß ebenso ständig Lohnkosten beglichen und ein Erlös realisiert werden. An der formalen Struktur der Produktionsgleichungen änderte sich somit nichts: Insofern hält der von Helmedag konstruierte Gegensatz zwischen einer Betrachtung von Stromgrößen und einer von Beständen einer differentialanalytischen Betrachtung nicht stand. Als mathematischer Ausdruck kontinuierlich erfolgender Zahlungen des Preises der Produkte, der Vorprodukte und der Löhne entgeht die Gleichung (i) andererseits dem Kurzschen Vorwurf der Widersprüchlichkeit. Dazu muß man allerdings von dem Aspekt der Zahlungsziele etc., der von Helmedag betont worden ist, um seine Gleichung plausibel zu machen, völlig absehen. Man hat schlicht und einfach von der (dann unbegründeten) Annahme auszugehen, daß sich Profiterwartungen lediglich an die Lohnkosten und ihren Umfang knüpfen. Dieser Ansatz muß nicht einmal eine dogmatische Setzung sein, wenn man nämlich bereit ist, die Folgen dieses theoretischen Ansatzes empirisch zu überprüfen.

\section{„Fixkosten“ sind kein Ersatz für eine exakte Kostenrechnung}

Nach dem Grenzübergang zu einer dynamischen Betrachtungsweise bleiben nach Helmedag die Zinsen für das ,,investierte eigene oder fremde Geld“52 übrig, die betriebswirtschaftlich als „Fixkosten“ in Erscheinung treten. „Zinsen für Fremdkapital bzw. die (nicht unbedingt auszahlungswirksamen) kalkulatorischen Eigenkapitalzinsen sind Fixkosten. Soll das Unternehmen Bestand haben, dürfen sie langfristig nicht über dem Deckungsbeitrag liegen. "53 Natürlich kann man alle ständig abfließenden Gelder, die die Kosten für die Nutzung der Produktionsmittel begleichen, unter den Titel Fixkosten zusammenfassen. Nichts ist auch gegen die Betrachtungsweise einzuwenden, daß die Fixkosten durch die laufenden Einnahmen ständig gedeckt werden müssen.

Wenn wir diesen Gedanken fortsetzen, dann werden wir logischerweise die Differenz zwischen den Stromgrößen Erlöse (Helmedags „Deckungsbeitrag“) und den laufenden Kosten (seien sie nun fix oder nicht) als (differentiellen) Gewinn bezeichnen. Die Relation dieses Gewinnes zu den Kosten bildet dann wieder die (differentielle) Profitrate, die für verschiedene Unternehmen unterschiedlich sein wird.

Der Vorschlag Helmedags, den Gewinn aufgrund des Arbeitseinsatzes zu verteilen, würde zu extrem niedrigen Profitraten oder gar zum Ruin bei denjenigen

52 Vgl. Helmedag, F., WmA, S. 249 f.

53 Ebenda, S. 321. 
Unternehmen führen, die einen relativ hohen Anteil an Fixkosten unter der Rubrik Material oder Investitionsgüter haben. Die entsprechende Argumentation ist bekannt. Sie unterscheidet sich nicht von der einer stationären Betrachtungsweise. Helmedag selbst formuliert mit dürren Worten die entsprechende conditio sine qua non: „Der den einzelnen Zweigen in Proportion zum Arbeitsaufwand zufließende Profit muß ausreichen, um die Kapitaleinlage der Eigentümer zu verzinsen." 54 Genau hier liegt ein wunder Punkt der Helmedagschen Begründung der Werttheorie. Selbst die hochstilisierte unternehmerische Perspektive kann nicht verhindern, daß im Hintergrund der Kapitalist die Fäden zieht. Oder etwas sachlicher ausgedrückt: Wenn der gesamtwirtschaftliche Gewinn den Zweigen in Proportion zum Arbeitsaufwand zufließt, dann kommen Zweige mit einer überdurchschnittlichen Kapitalintensität (hohe Proportion Kapital zu Arbeit) regelmäßig zu schlecht weg: im Extremfall reicht ihr Gewinnanteil eben nicht aus, um die Kapitaleinlage der Eigentümer zu verzinsen.

Wie man sieht: das Helmedag-Modell hat selbst einen grundlegenden Konstruktionsfehler: es kann die Existenz von Zweigen mit einer überdurchschnittlich hohen organischen Zusammensetzung (Kapitalintensität) nicht erklären.

\section{Verbliebene Aufgaben}

Abschließend sei es gestattet, einen Schritt zurückzutreten und aus unserer Sicht aufzulisten, welche Verluste und welche „Geländegewinne“ auf beiden Seiten des „Kampfplatzes“ gemacht worden sind. Sicher, die Helmedagsche Begründung der Werttheorie ist in wesentlichen Teilen zerstört worden. Dabei darf man aber nicht übersehen, daß nach einem weithin akzeptierten wissenschaftstheoretischen Verständnis Theorien überhaupt keiner Begründung bedürfen. Was man von ihnen verlangen können muß, ist, daß sie in sich widerspruchsfrei sind, daß sie empirisch relevante und überprüfbare Folgerungen erlauben und daß sie im Vergleich mit Theorien, die sich auf den gleichen Gegenstandsbereich beziehen, mehr erklären / voraussagen können als diese. Helmedags Verdienst ist u.E. mit dem ausführlichen Nachweis verbunden, daß die Werttheorie den Vergleich mit der neoricardianischen Theorie keinesfalls zu scheuen braucht. Das kann man allerdings erst richtig würdigen, wenn man gewisse, zum Teil längst bekannte Eigenschaften der Produktionspreistheorie Sraffas als Anomalien problematisiert - so wie Helmedag das tut. Etwas kurz kommen bei diesem Vergleich die von Helmedag so genannten Bortkiewicz-Preise weg. U. E. käme es bei der weiteren Forschung darauf an, die empirisch relevanten Unterschiede, zu denen die drei Modelle führen, explizit herauszuarbeiten, um so

54 Ebenda. 
gediegene empirische Arbeiten zu ermöglichen, die jene Unterschiede mit der ökonomischen Realität konfrontieren. Nur auf diese Weise kann eine wissenschaftlich verbindliche Entscheidung zwischen den diskutierten drei Preissystemen herbeigeführt werden. In diesem Prozeß könnte es dann auch eine Rolle spielen, ob man - wie Helmedag - von einer simplifizierten Version der Arbeitswerttheorie ausgeht oder ob man sich endlich dazu entschließt, die Heterogenität der Arbeit auch im mathematischen Modell zu berücksichtigen. 55

\section{Literatur}

Fees-Dörr,E. (1989), Die Redundanz der Mehrwerttheorie. Marburg.

Helmedag, F. (1992), Warenproduktion mittels Arbeit. Zur Rehabilitation des Wertgesetzes. Marburg.

Helmedag, F.(1993), Zur Berechtigung der grundlegenden theoretischen Konstruktion von Marx im ersten Band des „Kapital“. In: Jahrbücher für Nationalökonomie und Statistik, Bd. 212, S. 442-450.

Helmedag, F. (1995), Ohne Fleiß kein Preis: Nochmals zur Erklärungskraft der Arbeitswertlehre. In: Jahrbücher für Nationalökonomie und Statistik, Bd. 214, S. 470-482.

Kurz, H.D. (1995), F. Helmedag und die „ökonomische Logik“. In: Jahrbücher für Nationalökonomie und Statistik, Bd. 214, S. 710-727.

Lager, Ch. $\left(1995^{1}\right)$, Über einen weiteren vergeblichen Versuch zur Rechtfertigung der Arbeitswertlehre. Ein Kommentar zu Professor Helmedag. In: Jahrbücher für Nationalökonomie und Statistik, Bd. 214, S. 463-469.

Lager, Ch. $\left(1995^{2}\right)$ Auch mit viel Fleiß - kein Preis oder Einstimmigkeit ist nicht leicht zu erzielen. Eine Antwort auf Professor Helmedag. In: Jahrbücher für Nationalökonomie und Statistik, Bd. 214 (1995), S. 728-731.

Quaas, F. (1991), Wolfgang Mühlpfordt - ein Vorgänger von Bortkiewicz? Zu den theoretischen Quellen des sogenannten Transformationsproblems. In: Jahrbücher für Nationalökonomie und Statistik, Bd. 208, S. 493-504.

Quaas, F. (1992), Das Transformationsproblem. Marburg.

Quaas, G. (1987), Wert und Gebrauchswert als Strukturen im ökonomischen Reproduktionsprozeß einer warenproduzierenden Gesellschaft. In: Wissenschaftliche Zeitschrift der KarlMarx-Universität Leipzig, Gesellschaftswissenschaftliche Reihe, Bd. 36, S. 391-402.

Smirnow, W.I. (1973), Lehrgang der höheren Mathematik, Teil I, Berlin.

Woll, A. (1993), Allgemeine Volkswirtschaftslehre, 11. Aufl., München.

55 Vgl. dazu Quaas, G. (1987), Wert und Gebrauchswert als Strukturen im ökonomischen Reproduktionsprozeß einer warenproduzierenden Gesellschaft. In: Wissenschaftliche Zeitschrift der Karl-Marx-Universität Leipzig, Gesellschaftswissenschaftliche Reihe, Bd. 36, S. 394 ff. 